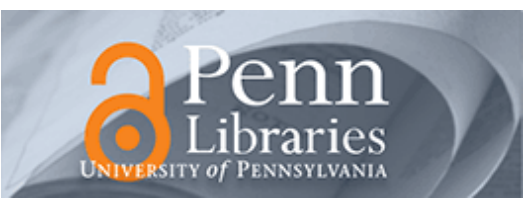

University of Pennsylvania ScholarlyCommons

$11-13-2006$

\title{
Graded Interface Models for More Accurate Determination of van der Waals-London Dispersion Interactions Across Grain Boundaries
}

\author{
Klaus van Benthem \\ Oak Ridge National Laboratory \\ Guolong Tan \\ University of Pennsylvania \\ Roger H. French \\ University of Pennsylvania; DuPont Company \\ Linda K. DeNoyer \\ Deconvolution and Entropy Consulting \\ Rudolf Podgornik

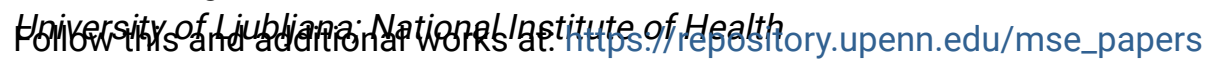 \\ Part of the Materials Science and Engineering Commons
}

See next page for additional authors

Recommended Citation

van Benthem, K., Tan, G., French, R. H., DeNoyer, L. K., Podgornik, R., \& Parsegian, V. A. (2006). Graded Interface Models for More Accurate Determination of van der Waals-London Dispersion Interactions Across Grain Boundaries. Retrieved from https://repository.upenn.edu/mse_papers/210

Suggested Citation:

van Benthem, K. et al. (2006). Graded interface models for more accurate determination of van der Waals-London dispersion interactions across grain boundaries. Physical Review B 74, 205110.

(C) 2006 American Physical Society

http://dx.doi.org/10.1103/PhysRevB.74.205110

This paper is posted at ScholarlyCommons. https://repository.upenn.edu/mse_papers/210

For more information, please contact repository@pobox.upenn.edu. 


\title{
Graded Interface Models for More Accurate Determination of van der Waals- London Dispersion Interactions Across Grain Boundaries
}

\begin{abstract}
Attractive van der Waals-London dispersion interactions between two half crystals arise from local physical property gradients within the interface layer separating the crystals. Hamaker coefficients and London dispersion energies were quantitatively determined for $\Sigma 5$ and near- $\Sigma 13$ grain boundaries in $\mathrm{SrTiO}_{3}$ by analysis of spatially resolved valence electron energy-loss spectroscopy (VEELS) data. From the experimental data, local complex dielectric functions were determined, from which optical properties can be locally analyzed. Both local electronic structures and optical properties revealed gradients within the grain boundary cores of both investigated interfaces. The results show that even in the presence of atomically structured grain boundary cores with widths of less than $1 \mathrm{~nm}$, optical properties have to be represented with gradual changes across the grain boundary structures to quantitatively reproduce accurate van der Waals-London dispersion interactions. London dispersion energies of the order of $10 \%$ of the apparent interface energies of $\mathrm{SrTiO}_{3}$ were observed, demonstrating their significance in the grain boundary formation process. The application of different models to represent optical property gradients shows that long-range van der Waals-London dispersion interactions scale significantly with local, i.e., atomic length scale property variations.
\end{abstract}

Disciplines

Engineering | Materials Science and Engineering

\section{Comments}

Suggested Citation:

van Benthem, K. et al. (2006). Graded interface models for more accurate determination of van der Waals-London dispersion interactions across grain boundaries. Physical Review B 74, 205110.

(C) 2006 American Physical Society

http://dx.doi.org/10.1103/PhysRevB.74.205110

\section{Author(s)}

Klaus van Benthem, Guolong Tan, Roger H. French, Linda K. DeNoyer, Rudolf Podgornik, and V. Adrian Parsegian 


\title{
Graded interface models for more accurate determination of van der Waals-London dispersion interactions across grain boundaries
}

\author{
Klaus van Benthem, ${ }^{1}$ Guolong Tan, ${ }^{2}$ Roger H. French, ${ }^{2,3}$ Linda K. DeNoyer, ${ }^{4}$ Rudolf Podgornik,,${ }^{5,6}$ and V. Adrian Parsegian ${ }^{6}$ \\ ${ }^{1}$ Oak Ridge National Laboratory, One Bethel Valley Road, Oak Ridge, Tennessee 37831-6031, USA \\ ${ }^{2}$ Materials Science Department, University of Pennsylvania, 3231 Walnut St,. Philadelphia, Pennyslvania 19104, USA \\ ${ }^{3}$ DuPont Company, Central Research, E356-384, Experimental Station, Wilmington, Delaware 19880-0356, USA \\ ${ }^{4}$ Deconvolution and Entropy Consulting, 755 Snyder Hill, Ithaca, New York 14850, USA \\ ${ }^{5}$ Department of Physics, University of Ljubljana, Jadranska 19, 1000 Ljubljana, Slovenija \\ ${ }^{6}$ Laboratory of Structural Biology, National Institute of Health, 9000 Rockville Pike, Bethesda, Maryland 20892, USA
}

(Received 1 June 2006; revised manuscript received 23 August 2006; published 13 November 2006)

\begin{abstract}
Attractive van der Waals-London dispersion interactions between two half crystals arise from local physical property gradients within the interface layer separating the crystals. Hamaker coefficients and London dispersion energies were quantitatively determined for $\Sigma 5$ and near- $\Sigma 13$ grain boundaries in $\mathrm{SrTiO}_{3}$ by analysis of spatially resolved valence electron energy-loss spectroscopy (VEELS) data. From the experimental data, local complex dielectric functions were determined, from which optical properties can be locally analyzed. Both local electronic structures and optical properties revealed gradients within the grain boundary cores of both investigated interfaces. The results show that even in the presence of atomically structured grain boundary cores with widths of less than $1 \mathrm{~nm}$, optical properties have to be represented with gradual changes across the grain boundary structures to quantitatively reproduce accurate van der Waals-London dispersion interactions. London dispersion energies of the order of $10 \%$ of the apparent interface energies of $\mathrm{SrTiO}_{3}$ were observed, demonstrating their significance in the grain boundary formation process. The application of different models to represent optical property gradients shows that long-range van der Waals-London dispersion interactions scale significantly with local, i.e., atomic length scale property variations.
\end{abstract}

DOI: 10.1103/PhysRevB.74.205110

PACS number(s): 78.20.Ci

\section{INTRODUCTION}

Most materials used for technological applications are polycrystalline. Their macroscopic properties are significantly influenced by the material's microstructure, especially by boundaries separating grains. General grain boundaries often consist of partially ordered, disordered, or even amorphous intergranular films ${ }^{1,2}$ whose composition can differ significantly from that within the adjacent grains ${ }^{3-7}$ due to, e.g., segregation effects. For this study we chose $\mathrm{SrTiO}_{3}$ as a model system for a functional ceramic that crystallizes into the cubic perovskite structure at room temperature. Polycrystalline perovskites have a wide range of applications, such as oxygen ion conductors in sensors, diverse electronic devices as varistors, ${ }^{8,9}$ piezoelectric materials in actuators, dielectric materials in capacitors, and as substrate for thin film growth of high $T_{c}$ superconductors. ${ }^{10}$ Examples where the macroscopic properties of these materials depend critically on the grain boundary structures and the intergranular films include increased toughness, ${ }^{11-13}$ reduced ionic conductivity, ${ }^{9}, 14-17$ diminished creep resistance, ${ }^{18,19}$ tunable electrical conductivity, ${ }^{20,21}$ decreased thermal conductivity, ${ }^{22}$ and enhanced sintering behavior. ${ }^{23}$ The reason for this dominance is that interfaces inherently contain a concentration of defects and dopants far in excess of the equilibrium distribution in the bulk of the material. ${ }^{24}$ This excess of defects results in the interface having a different atomic arrangement and consequently, different properties from the bulk. In addition, interfaces can serve as a source or sink of extrinsic segregant and dopant atoms that produce compositional variations at interfaces in addition to their structural variations.
In this study, we have used valence electron energy-loss spectroscopy (VEELS) in a scanning transmission electron microscope (STEM) (Refs. 25-32) to analyze the electronic band structure and the interband transition spectrum of a $\Sigma 5$ and a near- $\Sigma 13(\mathrm{n} \Sigma 13)$ grain boundary in $\mathrm{SrTiO}_{3}$. Interband transitions are analyzed quantitatively based on $a b$ initio calculated densities of states. ${ }^{33}$ Observed gradients in the interband transition strength for both grain boundary structures directly lead to locally modified optical properties within and close to the grain boundary cores and therefore to the appearance of appreciable van der Waals-London dispersion forces at the grain boundaries. The role of such long range interactions, which are a direct manifestation of quantum electrodynamics, has become a topic of critical interest in interface sciences. ${ }^{34-36}$

In this work we determine the van der Waals-London dispersion interactions using a new multilayer gradient method to represent more accurately gradual changes in the optical properties across the corresponding grain boundary cores. This directly leads to the determination of Hamaker coefficients. ${ }^{37,38}$ The magnitude of the attractive van der Waals-London dispersion force between two grains is expected to be very low for a dense, atomically abrupt interface like in the $\Sigma 5$ case, while for a boundary such as the $\mathrm{n} \Sigma 13$, the range of van der Waals-London dispersion forces can be appreciable and may play a role in the determination of equilibrium grain boundary thickness. ${ }^{32}$ These thermodynamic forces may drive segregation or depletion of the boundary as well as determine the adhesion strength of the interface. 


\section{EXPERIMENTAL DETAILS}

Two symmetrical tilt grain boundaries in $\mathrm{SrTiO}_{3}$ with a $\Sigma=5$ (310) $\theta=36.87^{\circ}$ and a near $\Sigma=13$ (510) $\theta=24^{\circ}$ orientation relationship, each formed by diffusion bonding of (0.14 wt. \%) Fe doped $\mathrm{SrTiO}_{3}$ single crystals, were prepared for cross-sectional TEM investigations following techniques described by Strecker et al. ${ }^{39}$ The bicrystals were cut, ground, dimpled, polished, and afterwards ion-beam thinned using $6 \mathrm{keV} \mathrm{Ar}^{+}$ions in a Gatan DUOMILL600 with an inclination angle of $12^{\circ}$. After perforation of the specimens, ion milling was continued at $3 \mathrm{keV}$ in order to remove most of damage layers done to the specimen surfaces. VEELS measurements were performed using a Gatan parallel electron energy-loss spectrometer (PEELS) attached to the Stuttgart VG Microscopes HB501 UX dedicated STEM. The beam current was reduced to less than $1 \mathrm{nA}$ in order to improve the energy resolution to better than $0.6 \mathrm{eV}$, measured by the full width at half maximum of the zero-loss peak. The electron probe size was about $0.5 \mathrm{~nm}$.

Spectra were acquired using an energy dispersion of $0.1 \mathrm{eV} /$ channel to record spectra up to energy-losses at higher than $90 \mathrm{eV}$. The convergence semiangle was chosen equal to the collection semiangle, which was given to $6.5 \mathrm{mrad}$ by the $2 \mathrm{~mm}$ PEELS entrance aperture. One hundred single spectra were acquired as line scans by scanning the electron probe perpendicular across the interface with lengths between 12.5 and $40 \mathrm{~nm}$. Therefore, the spatial separation of two adjacent spectra is between 0.125 and $0.4 \mathrm{~nm}$. Although probe sizes used are in the range of $0.4-0.6 \mathrm{~nm}$, this setup provides the determination of very local changes within the acquired spectra.

In order to decrease contamination effects in the STEM, the specimens were heat treated in the airlock of the microscope at approximately $100{ }^{\circ} \mathrm{C}$ for about $20 \mathrm{~min}$. By this procedure, carbon contamination effects during electron irradiation could be significantly reduced. Specimen thicknesses ranged from 1.5 to 2.5 multiples of inelastic mean free path to minimize influences of surface plasmons and remaining effects by contamination or beam damage due to the specimen preparation.

\section{DATA ANALYSIS}

For each energy-loss spectrum, the center of the zero-loss peak was determined, and the wings of the zero-loss peak were fitted by using a Pearson VII function up to a height varying between $20 \%$ and $40 \%$ of the maximum intensity. ${ }^{26}$ Subsequently the spectra were corrected for multiple scattering events by Fourier-logarithmic deconvolution ${ }^{40}$ and were scaled for constant relative thickness. ${ }^{31}$ For energies below $3.0 \mathrm{eV}$, i.e., close to the optical band gap energy of $\mathrm{SrTiO}_{3}$ (3.2 eV, ${ }^{41} 3.1 \mathrm{eV}$ Refs. 42-44), no transitions are expected. Therefore the intensity of the single-scattering spectrum below this value is set to zero. Cerenkov radiation was neglected, consistent with our previous observations. ${ }^{45}$ The single-scattering energy loss function (ELF), which is given by the double differential cross section $d^{2} \sigma / d E d \Omega$ for inelastic electron scattering, is proportional to $\operatorname{Im}-1 / \varepsilon(\omega)$, where $\varepsilon(\omega)=\varepsilon^{\prime}(\omega)+i \varepsilon^{\prime \prime}(\omega)$, is the complex dielectric function. ${ }^{40}$ $\varepsilon^{\prime}(\omega)$ and $\varepsilon^{\prime \prime}(\omega)$ are the real and the imaginary parts of the complex dielectric function, respectively. To be consistent with the language of measurement, from here on we use energy $E=\hbar \omega$ rather than the radial frequency $\omega$ as the independent parameter in the dielectric response function.

The real part of the inverse dielectric response function, $\operatorname{Re} \frac{1}{\varepsilon(E)}$, is obtained by a Kramers-Kronig transformation, described in detail elsewhere, ${ }^{26}$ which finally leads to the determination of the complex dielectric function from each single spectrum of the line scan. For a correct quantitative scaling of the amplitude of ELF, a scaling factor was determined using Eq. (1) based on a known value of $n=\sqrt{\varepsilon(0)}$ $=2.37$ for the refractive index within bulk $\mathrm{SrTiO}_{3}$ (Ref. 33)

$$
\begin{aligned}
1-\operatorname{Re}\left[\frac{1}{\varepsilon(0)}\right] & =\frac{2}{\pi} \int \operatorname{Im}\left[\frac{-1}{\varepsilon(E)}\right] \frac{d E}{E} \\
& =\frac{2}{\pi} \int[\text { Scale factor } * \text { Data }] \frac{d E}{E} .
\end{aligned}
$$

After calibrating ELF within the bulk material, the subsequent data sets can be quantitatively analyzed even within the grain boundary area.

Once the dielectric function is derived, the complex interband transition strength $J_{c v}$ can be calculated in the following form

$$
J_{c v}(E)=J_{c v}^{\prime}+i J_{c v}^{\prime \prime}=\frac{m_{0}^{2}}{e^{2} \hbar^{2}} \frac{E^{2}}{8 \pi^{2}}\left[\varepsilon^{\prime \prime}(E)+i \varepsilon^{\prime}(E)\right],
$$

where $m_{0}$ is the electron mass and $e$ its charge. $J_{c v}$ corresponds to the joint densities of states and has units of $\mathrm{g} \mathrm{cm}^{-3} \cdot 27$ For computational convenience, we take the prefactor in Eq. (2), whose value in cgs units is 8.289 $\times 10^{-6} \mathrm{~g} \mathrm{~cm}^{-3} \mathrm{eV}^{-2}$, as unity. Therefore, the units of $J_{c v}(E)$ spectra become $\mathrm{eV}^{2}$.

The analysis of the spectra was done using electronic structure tools (EST). ${ }^{46}$ The effective number of electrons per cubic centimeter, $n_{e f f}$, contributing to interband transitions up to energy $E$, is calculated using the oscillator strength or $f$-sum rule, ${ }^{47}$ shown in Eq. (3), evaluated for $J_{c v}^{\prime}$ as

$$
n_{e f f}(E)=\frac{4}{m_{0}} \int_{0}^{E} \frac{J_{c v}^{\prime}\left(E^{\prime}\right)}{E^{\prime}} d E^{\prime}=\frac{m_{0}}{2 \pi^{2} \hbar^{2} e^{2}} \int_{0}^{E} E^{\prime} \varepsilon_{2} d E^{\prime}
$$

Optical properties are commonly formulated in the cgs unit system. However, the reported results from $f$-sum rule calculations below are converted to electrons per cubic nanometer.

\section{ATOMIC AND ELECTRONIC STRUCTURE RESULTS}

\section{A. Interband transition strength}

Figure 1 shows three-dimensional (3D) plots of the interband transition strength $\operatorname{Re}\left[J_{c v}\right]$ for the $\Sigma 5$ [Fig. 1(a)] and the $n \Sigma 13$ [Fig. 1(b)] grain boundaries as a function of energy and relative position. Intensities are color coded. Intensity 

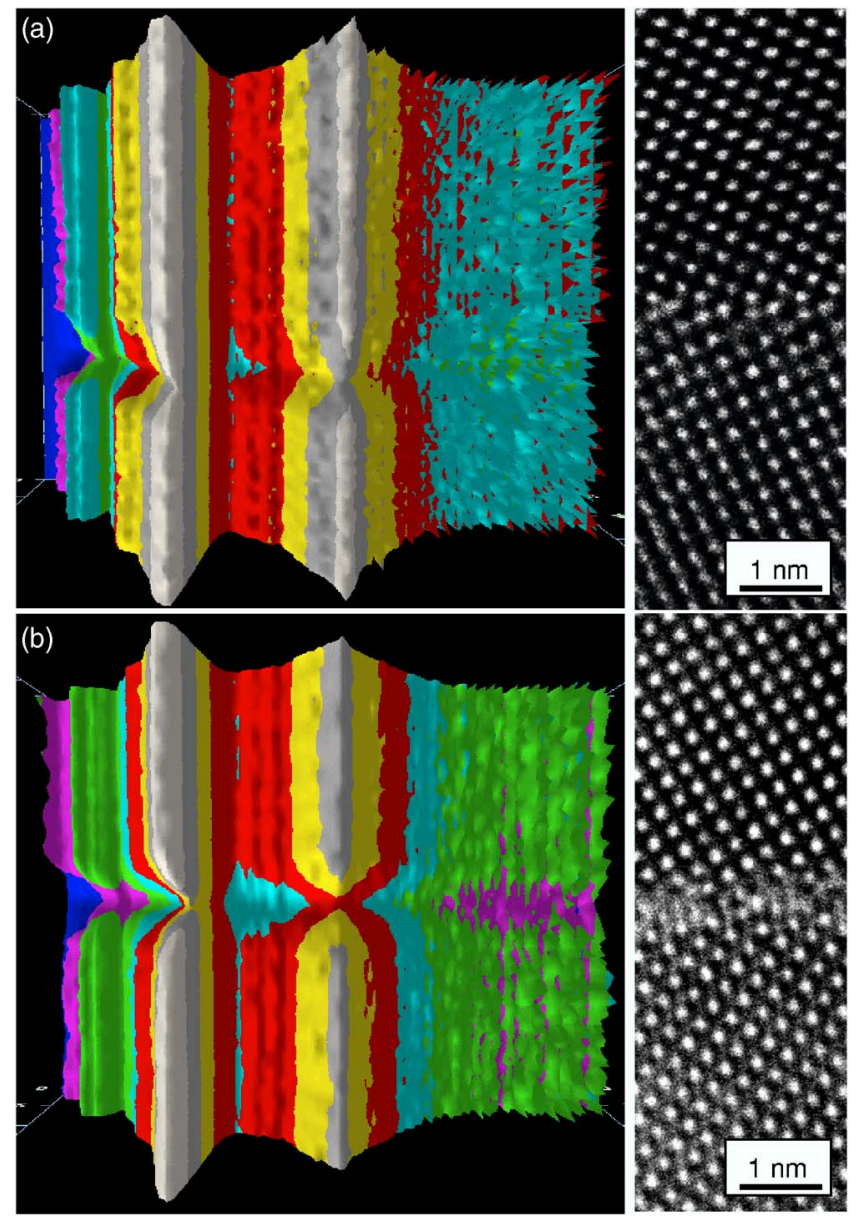

FIG. 1. (Color online) 3D representation of the interband transition strength spectrum across both the $\Sigma 5$ (a) and the $n \Sigma 13$ (b) grain boundaries (not to scale). Intensities are color coded. Both data sets show significant dips where the GB cores are located. The HRTEM images show that both interfaces show atomically structured grain boundary cores.

variations within the data are no longer related to any thickness variations of the TEM specimens. Instead, changes in intensity arise directly from local changes in the quantitative optical properties and electronic structure across the interfaces. For each grain boundary a high-resolution transmission electron microscopy (HRTEM) image is shown. Kienzle et $a l^{48}$ and Hutt et al. ${ }^{49}$ have demonstrated that both grain boundaries have atomically structured grain boundary cores and do not show intergranular films. However, the $\mathrm{n} \Sigma 13$ grain boundary structure appears less dense compared to the $\Sigma 5$ case and is characterized by a smaller structural periodicity along the interface plane.

The interband transition strength exhibits a large decrease in amplitude across both interfaces, as observed in the centers of each GB core. Spectra well apart from the GB cores show exactly the same line shapes for the front and the back grain. An assignment of transitions in the densities of states to peaks seen in the interband transition strengths allow an understanding of these changes on the basis of changes in the local electronic structure and bonding at the interfaces. To analyze this in further detail, three single $\operatorname{Re}\left[J_{c v}\right]$ spectra,

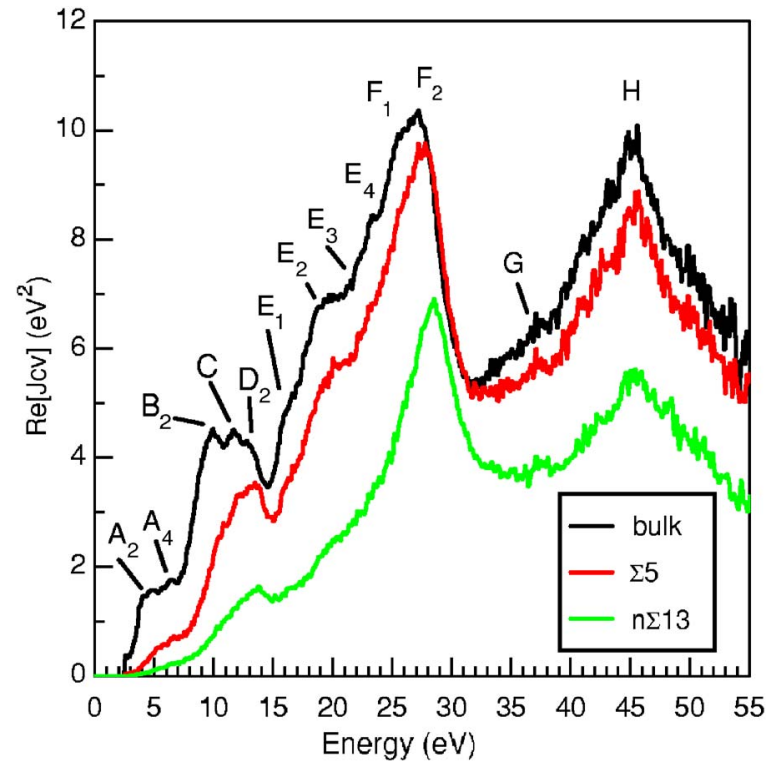

FIG. 2. (Color online) Interband transition strength spectra recorded from bulk $\mathrm{SrTiO}_{3}$ as well as from the $\Sigma 5$ and the $\mathrm{n} \Sigma 13$ grain boundary cores. Labeled transitions are assigned in Table I.

two extracted from each GB core together with one from bulk $\mathrm{SrTiO}_{3}$ are plotted in Fig. 2. It is observed that over the entire spectrum, the interband transition strength is strongest in bulk $\mathrm{SrTiO}_{3}$, followed by the spectra for the $\Sigma 5$ and the $\mathrm{n} \Sigma 13$ grain boundaries. Featured peaks in Fig. 2 are labeled $\mathrm{A}_{2}-\mathrm{H}$, which correspond to different interband transitions for both grains and boundaries. Differences between the bulk $\mathrm{SrTiO}_{3}$ material and the two GBs are summarized in Table I. In the low-energy region below $8 \mathrm{eV}$ no significant features, except for a small remnant of $A_{2}-A_{4}$ remain evident in the $\mathrm{n} \Sigma 13$ interface, but some intensity of the $A_{2}-A_{4}$ peaks is present for the $\Sigma 5$ interface in Fig. 2. The triplet structure $B-C-D$ observed in bulk $\mathrm{SrTiO}_{3}$ has depressed strength in the two boundary cores with the $\mathrm{n} \Sigma 13$ interface again showing the greatest decrease in intensity. Peaks $B$ and $C$ exhibit smaller intensities in the grain boundary core region than in the bulk. At $13.6 \mathrm{eV}$, a new shoulderlike-peak labeled $D^{*}$ appears at both the $\Sigma 5$ and the $n \Sigma 13$ boundary, which may be a interpreted as a new interface specific feature or as just the bulk transition labeled $D$ shifted towards higher energy. Transitions $E_{2}$ and $E_{1}$ at $20.1 \mathrm{eV}$ and $16.5 \mathrm{eV}$ are reduced in intensity while peak $E_{2}$ remains distinct in the $\Sigma 5$ grain boundary structure. The weak features labeled $E_{3}$ and $E_{4}$ are not observable at either of the two grain boundaries. The most intense transition labeled $F_{2}$ at $27.3 \mathrm{eV}$ shifts by $1.1 \mathrm{eV}$ to higher energy whereas the slight shoulder $F_{1}$ at $26.1 \mathrm{eV}$ disappears in both grain boundary core structures. The weak features labeled $G$ at $37.5 \mathrm{eV}$ is not observable above the noise limit inside the GB cores while strong peak at $45 \mathrm{eV}$ labeled $H$ is present in all three spectra in Fig. 2.

\section{B. Interfacial electron density}

Following Eq. (3) oscillator strength $f$-sum rule calculations were performed for the two investigated GBs in com- 
TABLE I. Interband transition energies (in eV) for bulk $\mathrm{SrTiO}_{3}$ the $\Sigma 5$ and the $\mathrm{n} \Sigma 13$ grain boundary as determined from spatially resolved VEELS (see Fig. 2). Peak assignments are based on total densities of states analyses (see Ref. 33). Transitions are labeled as in Fig. 2 with $p$ denoting a peak and s a shoulder.

\begin{tabular}{|c|c|c|c|c|c|}
\hline Peaks & Assignment & Type & Bulk & $\Sigma 5 \mathrm{~GB}$ & $\mathrm{n} \Sigma 13 \mathrm{~GB}$ \\
\hline \multicolumn{6}{|c|}{ Transitions From the $\mathrm{O} 2 p$ Upper Valence Band } \\
\hline$A_{1}$ & $\mathrm{O} 2 p \Rightarrow \mathrm{Ti} 3 d t_{2 g}$ & $s$ & 4.2 & $* * *$ & $* * *$ \\
\hline$A_{2}$ & $\mathrm{O} 2 p \Rightarrow \operatorname{Ti} 3 d t_{2 g}$ & $p$ & 4.8 & $* * *$ & $* * *$ \\
\hline$A_{3}$ & $\mathrm{O} 2 p \Rightarrow \operatorname{Ti} 3 d t_{2 g}$ & $s$ & 5.3 & $* * *$ & $* * *$ \\
\hline$A_{4}$ & $\mathrm{O} 2 p \Rightarrow \operatorname{Ti} 3 d t_{2 g}$ & $p$ & 6.3 & 7 & $* * *$ \\
\hline$B_{1}$ & $\mathrm{O} 2 p \Rightarrow \operatorname{Ti} 3 d e_{g}$ & $p$ & 9.1 & $* * *$ & $* * *$ \\
\hline$B_{2}$ & $\mathrm{O} 2 p \Rightarrow \operatorname{Ti} 3 d e_{g}$ & $p$ & 9.9 & $* * *$ & $* * *$ \\
\hline$C$ & $\mathrm{O} 2 p \Rightarrow \operatorname{Ti} 3 d e_{g}, \operatorname{Sr} 4 d t_{2 g}$ & $p$ & 11.9 & $* * *$ & $* * *$ \\
\hline$D_{1}$ & $\mathrm{O} 2 p \Rightarrow \mathrm{Sr} 4 d t_{2 g}$ & $s$ & 12.9 & $* * *$ & $* * *$ \\
\hline$D_{2}$ & $\mathrm{O} 2 p \Rightarrow \mathrm{Sr} 4 d t_{2 g}$ & $s$ & 13.7 & 14 & 13.6 \\
\hline \multicolumn{6}{|c|}{ Transitions predominantly from the $\mathrm{O} 2 s, \mathrm{Sr} 4 p$ lower valence band } \\
\hline$E_{1}$ & $\operatorname{Sr} 4 p \Rightarrow \operatorname{Ti} 3 d t_{2 g}$ & $s$ & 16.4 & $* * *$ & 16.5 \\
\hline$E_{2}$ & $\operatorname{Sr} 4 p \Rightarrow \operatorname{Ti} 3 d e_{g}$ & $s$ & 19.7 & $* * *$ & 20.2 \\
\hline$E_{3}$ & $\operatorname{Sr} 4 p \Rightarrow \operatorname{Sr} 4 d t_{2 g}$ & $s$ & 21.7 & $* * *$ & $* * *$ \\
\hline$E_{4}$ & $\mathrm{Sr} 4 p \Rightarrow \mathrm{Sr} 4 d e_{g}$ & $s$ & 24.2 & $* * *$ & $* * *$ \\
\hline$F_{1}$ & $\mathrm{O} 2 s \Rightarrow \mathrm{Ti} 3 d, \mathrm{Sr} 4 d$ & $s$ & 26.4 & $* * *$ & $* * *$ \\
\hline$F_{2}$ & $\mathrm{O} 2 s \Rightarrow \mathrm{Ti} 3 d, \mathrm{Sr} 4 d$ & $p$ & 27.4 & 27.7 & 28.4 \\
\hline \multicolumn{6}{|c|}{ Transitions predominantly from the $\mathrm{Sr} 4 s$, Ti $3 p$ shallow core level } \\
\hline$G$ & $\operatorname{Sr} 4 s$, Ti $3 p \Rightarrow$ Ti $3 \mathrm{~d} t_{2 g}$ & $s$ & 37.5 & $* * *$ & 37.5 \\
\hline$H$ & $\begin{array}{c}\text { Ti } M_{2,3} \text { Edge } \\
\text { Sr } 4 s, \text { Ti } 3 p \Rightarrow \operatorname{Ti~} 3 d \text { Sr } 4 d \Rightarrow \\
\operatorname{Ti~} 3 d, \operatorname{Ti} 4 s, \text { Sr } 4 d\end{array}$ & $p$ & 45.5 & 45.0 & 45.5 \\
\hline
\end{tabular}

parison to bulk $\mathrm{SrTiO}_{3} \cdot{ }^{32}$ The number of valence electrons per cubic nanometer undergoing interband transitions was evaluated up to a transition energy of $90 \mathrm{eV}$. Although the $f$-sum rule data are qualitatively comparable in all three cases, reduced electron densities were observed for both grain boundary structures when compared to the bulk. In bulk $\mathrm{SrTiO}_{3}$, a total valence electron count of $558 \mathrm{e} / \mathrm{nm}^{3}$ at $90 \mathrm{eV}$ is reached, while for the $\Sigma 5$ GB only about $428 \mathrm{e} / \mathrm{nm}^{3}$ have undergone interband transitions by $90 \mathrm{eV}$. At the $\mathrm{n \Sigma} 13 \mathrm{~GB}$ an even lower value of $234 \mathrm{e} / \mathrm{nm}^{3}$ was observed. For the entire spectral range, the valence electron count for the $\Sigma 5$ boundary lies in between those for bulk $\mathrm{SrTiO}_{3}$ and the $\mathrm{n} \Sigma 13$ boundary.

\section{Index of refraction}

The index of refraction $n$ was evaluated as a function of

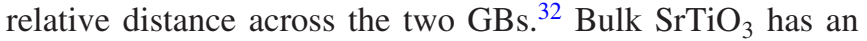
index of refraction of $2.37,{ }^{33}$ which drops significantly to about $1.56 \pm 0.05$ and $1.29 \pm 0.05$ for the $\Sigma 5$ and the $n \Sigma 13$ GB, respectively.

\section{CALCULATION OF HAMAKER COEFFICIENTS FOR COMPLEX CONFIGURATIONS}

The interfacial physical properties vary across an atomically structured GB, and they exhibit local physical properties which distinguish them from bulk phases. The significant decrease in the local index of refraction as well as the electron density in the GB core, compared to the bulk, demonstrate the presence of strong optical property gradients. ${ }^{31} \mathrm{Be}-$ cause of the presence of these gradients, which are the fundamental basis of the long-range van der Waals-London dispersion interaction, ${ }^{37}$ it becomes feasible to determine London dispersion forces and interaction energies and their role in interface formation and energy.

Once the electronic structure of bulk $\mathrm{SrTiO}_{3}$ and of the interface has been determined, the full spectral Hamaker coefficient, ${ }^{37}$ which sets the magnitude of the van der WaalsLondon attraction between two grains, can be calculated using the Lifshitz theory. ${ }^{50,51}$ In the following, we describe two different approaches to determine Hamaker coefficients for GB, based on either a three layer model or a multiple layer model to represent detailed GB dielectric properties.

\section{A. Three layer Hamaker coefficients}

For the case of two grains of material 1 (label $A$ ) which are separated from each other by one unique intervening material 2 (label $B$ ) of thickness $L$ [cf. Fig. 3(a)], what we refer to as the three layer (121) configuration, the nonretarded Hamaker coefficient, $A_{121}^{N R}$, can be calculated following Ninham and Parsegian ${ }^{52}$ as

$$
A_{121}^{N R}=-12 \pi L^{2} G_{\mathrm{vdW}}(L) .
$$

$\mathrm{G}_{\mathrm{vdW}}(L)$ is the van der Waals-London dispersion interaction free energy per unit surface area in the nonretarded limit of 


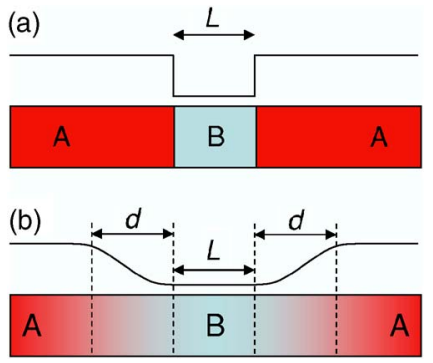

FIG. 3. (Color online) Sketches of an abrupt grain boundary (a) with an intergranular material $B$ between two bulk grains of material $A$. (b) represents a graded interface with gradual property changes between the grains and the boundary core. $L$ denotes the width of the grain boundary core perpendicular to the interface plane.

small interlayer separations. Although retardation effects due to the finite velocity of light are small at the small separations considered here, ${ }^{37,53}$ they are nevertheless included in the computational programs. Mathematical details for the formulation of the van der Waals interactions with incorporated retardation effects are omitted here and are published elsewhere. ${ }^{37,52,54} \mathrm{G}_{\mathrm{vdW}}(L)$ can be obtained from Eq. (5) (Ref. 53).

$$
G_{\mathrm{vdW}}(L)=\frac{\hbar}{4 \pi^{2}} \int_{0}^{\infty} \rho d \rho \int_{0}^{\infty} \ln D_{121}(L ; \xi, \rho) d \xi
$$

The secular determinant of electromagnetic field modes $D_{121}(\xi, \rho)$ is obtained again in the nonretarded limit from ${ }^{52,53}$

$$
D_{121}(L ; \xi, \rho)=1-\Delta_{12}^{2}(i \xi) e^{-2 L \rho} .
$$

$\Delta_{12}(i \xi)$ represents the difference of the London dispersion spectra on the two sides of an interface between materials 1 and 2 evaluated at imaginary frequencies, given by

$$
\Delta_{12}(i \xi)=\frac{\varepsilon_{1}(i \xi)-\varepsilon_{2}(i \xi)}{\varepsilon_{1}(i \xi)+\varepsilon_{2}(i \xi)} .
$$

One should note here that the magnetic properties of the layers are considered to be homogeneous and thus irrelevant for the evaluation of the layer eigenmodes. The London dispersion spectra $\varepsilon(i \xi)$ are obtained from the imaginary part of the dielectric constant $\varepsilon^{\prime \prime}(E)$ as the Kramers-Kronig transform ${ }^{37,55}$

$$
\varepsilon(i \xi)=1+\frac{2}{\pi} \int_{0}^{\infty} \frac{(E / \hbar) \varepsilon^{\prime \prime}(E / \hbar)}{(E / \hbar)^{2}+\xi^{2}} d(E / \hbar) .
$$

In this three-layer system, the interfacial material serves to shield the attraction of the two $\mathrm{SrTiO}_{3}$ grains. The Hamaker coefficient is fictitiously infinite for a vacuum interlayer and zero if the interfacial material 2 is identical to material 1 .

However, a simple three-layer configuration does not adequately describe the experimental situation. Experimentally measured dielectric properties point to a quasi-continuous variation of the dielectric properties through the $\Sigma 5$ and $\mathrm{n} \Sigma 13$ interlayers [cf. Fig. 3(b)].

\section{B. Multilayer Hamaker coefficients}

In order to achieve a better representation of graded optical properties across even atomically abrupt grain boundaries, we introduce another approach to calculate Hamaker coefficient of the van der Waals-London dispersion interaction. The new concept is based on a finer, i.e., more than three, intergranular layer division. Instead of considering the three-layer 121 model, we divide the single intergranular layer into a series of multiple layers, such as 12321 or even 1234321 , to describe the graded properties across the interface. The limit of infinitely many subdivisions would represent a continuous distribution of dielectric properties across the intergranular space.

Hamaker coefficients for multilayer configurations are most easily calculated using a method described by Parsegian, ${ }^{53}$ in which one starts from a three layer 121 configuration and then systematically adds new layers between material 1 and 2, or between material 2 and 3, and so fourth, modifying the effective dielectric discontinuities at the interfaces that bound the central layer. For this reason we call this method add-a-layer. ${ }^{54}$ With a large number of subdivisions this approach can get as close to a quasicontinuous spatial distribution of dielectric properties as desirable to realistically describe the properties of the investigated grain boundary.

The Hamaker coefficient for a multilayer system described via a configurational mnemonic 12321, being the first higher order approximation when compared to the original 121 configuration, is obtained by generalizing Eq. (6), valid for the 121 configuration to the 12321 configuration. In this case the secular determinant of the EM field modes can be written in a similar form ${ }^{53}$

$$
D_{12321}(L ; \xi, \rho)=1-\Delta_{32}(i \xi) \Delta_{12}^{e f f}(d ; i \xi, \rho) e^{-2 \rho L} .
$$

$\Delta_{12}$ has been replaced by $\Delta_{12}^{e f f}(d ; i \xi, \rho)$, which can be shown to be of the form ${ }^{53}$

$$
\Delta_{12}^{e f f}(d ; i \xi, \rho)=\frac{\Delta_{13}(i \xi) e^{-\rho d}+\Delta_{32}(i \xi)}{1+\Delta_{13}(i \xi) \Delta_{32}(i \xi) e^{-\rho d}} .
$$

In Eqs. (9) and (10), $d$ is the thickness of layer 2, and $L$ is the thickness of the central layer 3. By adding layer after layer and constructing the corresponding secular determinant of the EM field modes at each step as detailed above, the adda-layer method allows us to compute Hamaker coefficients for any number of layers, generalizing Eq. (9) to a multilayer geometry.

\section{Special layers for use in Hamaker coefficient calculations}

Hamaker coefficients calculated for multilayer configurations are often very different in magnitude from those calculated for simple three layer systems. The presence of quasicontinuous gradients in dielectric properties reduces the magnitude of the attraction that is found for atomically abrupt interfaces.

In order to be able to calculate Hamaker coefficients with quasicontinuous gradients in the dielectric properties, most specifically in examples where gradient spectra do not exist, 
a mixture formulation was set up to approximate the effects of measured dielectric response gradients. The general approach in the mixture method is to assume a model form for the graded dielectric response, instead of the one experimentally determined, to calculate the appropriate Hamaker coefficients.

For this mixture layer, one needs to obtain $\varepsilon$, and thereby $\varepsilon(i \xi)$, using an approach such as the Bruggemann effective medium approximation ${ }^{56}$ which uses the full dielectric properties of the two materials in the mixture, i.e., the grain with $\varepsilon_{A}$ and the interface core with $\varepsilon_{B}$. Here, we use a simple volume average to obtain the London dispersion spectrum of $\varepsilon_{A+B}$, a mixture consisting of volume fractions $f_{A}(x)$ of material $A$ plus $f_{B}(x)$ of material $B$, with $f_{A}(x)+f_{B}(x)=1$. In this formulation, $f_{A}(x)$ is a function of the transverse coordinate $x$ perpendicular to the intergranular layer

$$
\begin{aligned}
\varepsilon_{A+B}(x ; i \xi)= & f_{A}(x) \varepsilon_{A}(i \xi)+f_{B}(x) \varepsilon_{B}(i \xi)=f_{A}(x) \varepsilon_{A}(i \xi) \\
& +\left(1-f_{A}(x)\right) \varepsilon_{B}(i \xi) .
\end{aligned}
$$

Though other algebraic mixture rules can be implemented, ${ }^{57}$ the Bruggemann effective medium approximation is the simplest scheme that can be implemented computationally. The form of this functional dependence specifies the nature of the model in the mixture formulation. Several different gradient templates, i.e., several forms of the functional dependence $f_{A}(x)$ were tested in this study and are described in the following.

We consider only symmetric cases where we measure the transverse position from $x=0$ at the plane of symmetry. The thickness of the variable region is always designated by $d$ [cf. Figs. 3(b), 4(a), and 4(b)]. Gradients are specified only for the right (positive $x$ ) side of the separating layer. All the different interlayers were described numerically by using 10 sublayers of equal thickness.

\section{Linear gradient layer}

For a linear gradient layer model, the fraction $f_{A}(x)$ at a distance $x$ into a layer of material $A$ of thickness $d$ varies linearly (see Fig. 4). It has the analytical form

$$
f_{A}(x)=\frac{1}{d}\left(x-\frac{L}{2}\right), \quad \frac{L}{2} \leqslant x \leqslant \frac{L}{2}+d .
$$

Note that linear gradient layers contain discontinuities in the gradient slope at the two sides of the layer. In realistic graded layers, the changes in material properties are more likely to be continuous in the value as well as in the derivative, as one moves into the layer. The corresponding volume fraction $f_{A}(x)$ should therefore be given within a different model scheme that we detail below and that we refer to as the "quadroid," the "circuloid," and the "quadratic" gradient models.

\section{Quadroid gradient layer}

A quadroid gradient layer model, composed of two parabolic arcs, provides a continuous change (in the value as well as the derivative) of the dielectric response from the grain with $\varepsilon_{A}$ into the interlayer core with $\varepsilon_{B}$ (Fig. 4). The equation
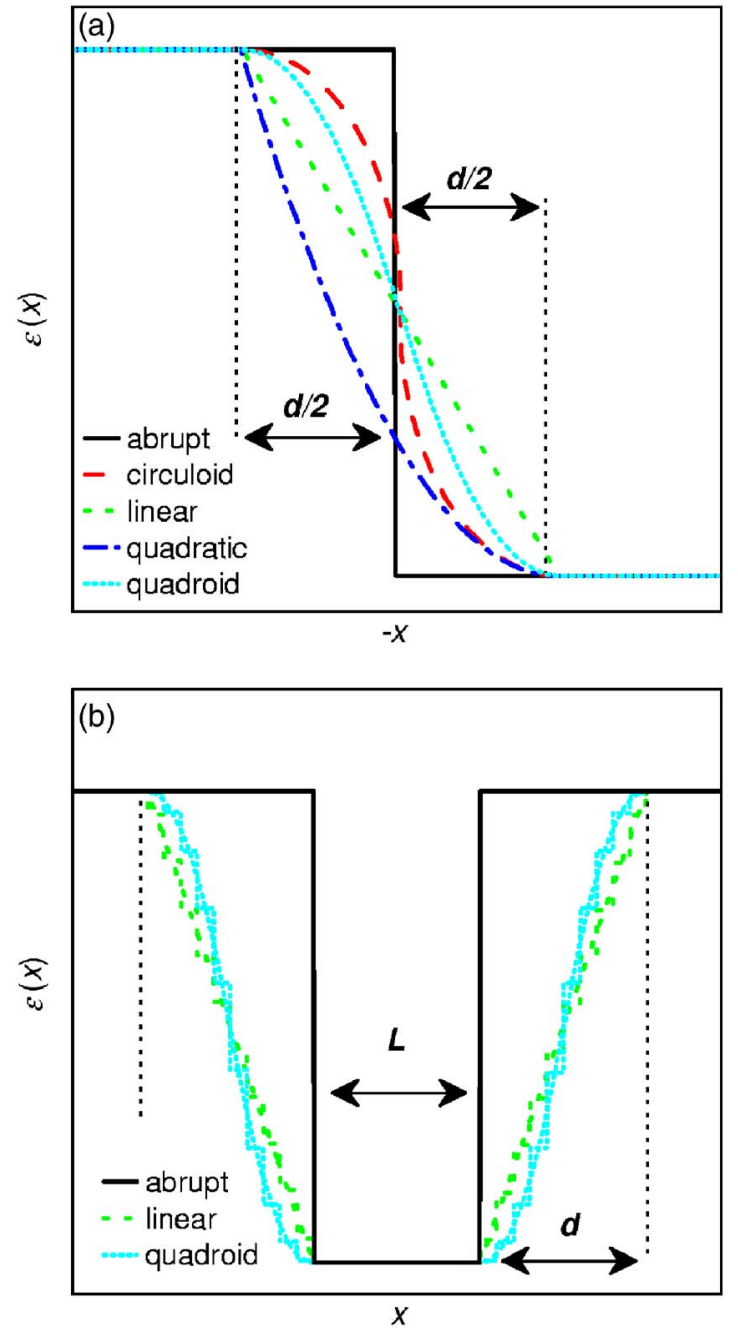

FIG. 4. (Color online) (a) sketches of various gradient layers (quadratic, quadroid, circuloid) as well as abrupt and linear layer models for the calculation of Hamaker coefficients for the different grain boundary structures. (b) shows the abrupt, the linear and the quadroid gradient models in more detail for the numerical analysis. The slopes of the gradients were approximated by 10 distinct steps of equal width, as it is shown for the linear and the quadroid models.

for the mixing fraction $f_{A}(x)$, at a distance $x$ into a quadroid layer of thickness $d$ has the form

$$
\begin{gathered}
f_{A}(x)=\frac{2}{d^{2}}\left(x-\frac{L}{2}\right)^{2}, \quad \frac{L}{2} \leqslant x \leqslant \frac{L}{2}+\frac{d}{2} \\
f_{A}(x)=1-\frac{2}{d^{2}}\left(x-\frac{L}{2}-d\right)^{2}, \quad \frac{L}{2}+\frac{d}{2} \leqslant x \leqslant \frac{L}{2}+d .
\end{gathered}
$$

\section{Circuloid gradient layer}

The circuloid gradient layer model is composed of two inverted circular arcs and provides a continuously varying dielectric response function from the grain into the interlayer (Fig. 4). In this case, $f_{A}(x)$ is given by 
TABLE II. Hamaker constants $(z J)$ as determined from the abrupt and the different graded interface layer models.

\begin{tabular}{cccccc}
\hline \hline Ham. coeff. & Abrupt & Linear & Quadratic & Circuloid & Quadroid \\
\hline $\mathrm{SrTiO}_{3}|\mathrm{Vac}.| \mathrm{SrTiO}_{3}$ & 243.9 & 176.4 & 151.5 & 164.0 & 171.2 \\
$\mathrm{SrTiO}_{3}|\mathrm{n} \Sigma 13| \mathrm{SrTiO}_{3}$ & 105.5 & 74.3 & 64.0 & 70.2 & 72.5 \\
$\mathrm{SrTiO}_{3}|\Sigma 5| \mathrm{SrTiO}_{3}$ & 35.0 & 21.3 & 21 & 20.0 & 20.7 \\
$\mathrm{SrTiO}_{3}\left|\mathrm{SiO}_{2}\right| \mathrm{SrTiO}_{3}$ & 68.2 & 48.5 & 42.0 & 49.0 & 50.3 \\
\hline \hline
\end{tabular}

$$
\begin{gathered}
f_{A}(x)=\frac{1}{2}-\frac{1}{d} \sqrt{\left(\frac{d}{2}\right)^{2}-\left(x-\frac{L}{2}\right)^{2}}, \quad \frac{L}{2} \leqslant x \leqslant \frac{L}{2}+\frac{d}{2} \\
f_{A}(x)=\frac{1}{2}+\frac{1}{d} \sqrt{\left(\frac{d}{2}\right)^{2}-\left(x-\frac{L}{2}-d\right)^{2}}, \quad \frac{L}{2}+\frac{d}{2} \leqslant x \leqslant \frac{L}{2}+d .
\end{gathered}
$$

\section{Quadratic gradient layer}

In the quadratic gradient layer model (Fig. 4) the volume fraction $f_{A}(x)$ of the intergranular space of thickness $d$ is now expressed via two inverted quadratic arcs as

$$
f_{A}(x)=\frac{1}{d^{2}}\left(x-\frac{L}{2}\right)^{2}, \quad \frac{L}{2} \leqslant x \leqslant \frac{L}{2}+d .
$$

\section{Results: van der Waals-London dispersion forces and Hamaker coefficients}

The Hamaker coefficients for both grain boundary structures were calculated using the five different approaches as explained above. Core widths perpendicular to the GB plane of 0.6 (Refs. 9 and 58) and 0.9 nm (Refs. 48 and 49) were used for the $\Sigma 5$ and the $n \Sigma 13$ grain boundaries, respectively. A region adjacent to the boundary core was chosen, in which quadratic, circuloid and quadroid gradient model features of the complex optical properties describe the quasicontinuous transition between the bulk grains and the boundary core, with a thickness of $d=0.195 \mathrm{~nm}$, corresponding to a Ti-O bond length in $\mathrm{SrTiO}_{3}$. Resulting Hamaker coefficients for the abrupt, linear, quadratic, circuloid, and quadroid interface models are listed in Table II and plotted in Fig. 5. In the "abrupt" case rather larger Hamaker constants were found, while all other cases reveal significantly smaller and almost identical values.

In relation to infinite separation, the change in Gibbs free energy per unit area of van der Waals-London dispersion interactions $G_{\mathrm{vdW}}(L)$ for a film of material 2 of finite thickness $L$ between two semi-infinite regions of material 1 is given by 59

$$
G_{\mathrm{vdW}}(L)=-\frac{A_{121}(L)}{12 \pi L^{2}} .
$$

A corresponding form of the Gibbs free energy per unit area for van der Waals-London dispersion interactions in multilayer geometries can be obtained with the add-a-layer method as described in Sec. IV B. In the numerical implementation retardation effects were considered to compute $G_{\mathrm{vdW}}(L)$. However, for the small intergranular separations, retardation effects are negligible. The resulting van der Waals-London dispersion interaction energies for the given core thicknesses are listed in Table III. As it was observed for the Hamaker constants, also the London dispersion energies are large for the abrupt interface description, while for the other cases rather comparable values were found.

\section{DISCUSSION}

\section{A. Electronic structure}

Every peak or shoulder in the interband transition strength data shown in Fig. 2 corresponds to one are more distinct transitions from certain initial states in the valence bands to certain final states in the conduction band. In an earlier study, we had done such peak assignments for $\operatorname{Re}\left[J_{c v}\right]$ data recorded from bulk $\mathrm{SrTiO}_{3} \cdot{ }^{33}$ The interband transition strengths for the $\Sigma 5$ and the $n \Sigma 13$ grain boundary structures will be analyzed and discussed in the following based on those assignments.

The shoulderlike transition $A_{4}$ at the $\Sigma 5 \mathrm{~GB}$ originates from excitations of $\mathrm{O} 2 p$ electrons into the $\mathrm{Ti} 3 d t_{2 g}$ band,

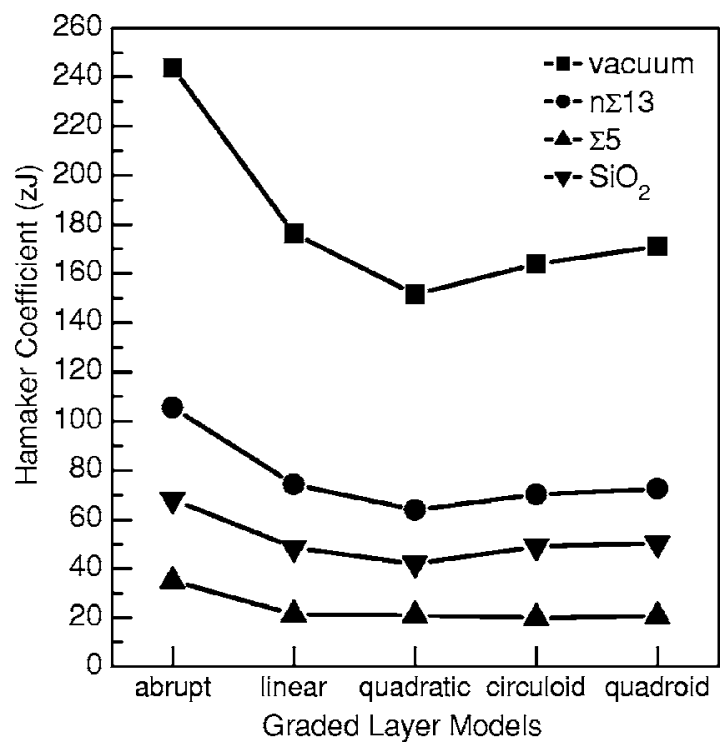

FIG. 5. Results from the Hamaker coefficient calculations based on the five different layer models for the two investigated grain boundary structures, and for a $\mathrm{SiO}_{2}$ and a vacuum interlayer between two $\mathrm{SrTiO}_{3}$ grain for comparison. 
TABLE III. London dispersion energies $\left(\mathrm{mJ} / \mathrm{m}^{2}\right)$ as determined using the abrupt and the different graded interface layer models.

\begin{tabular}{cccccc}
\hline \hline Disp. energy & Abrupt & Linear & Quadratic & Circuloid & Quadroid \\
\hline $\mathrm{SrTiO}_{3}|\mathrm{Vac}.| \mathrm{SrTiO}_{3}$ & 169 & 122.7 & 105.4 & 114.1 & 119.1 \\
$\mathrm{SrTiO}_{3}|\mathrm{n} \Sigma 13| \mathrm{SrTiO}_{3}$ & 73 & 51.7 & 44.5 & 48.8 & 50.4 \\
$\mathrm{SrTiO}_{3}|\Sigma 5| \mathrm{SrTiO}_{3}$ & 24 & 14.8 & 14.6 & 13.9 & 14.4 \\
$\mathrm{SrTiO}_{3}\left|\mathrm{SiO}_{2}\right| \mathrm{SrTiO}_{3}$ & 66 & 47.3 & 40.9 & 47.7 & 49.0 \\
\hline \hline
\end{tabular}

which are dramatically reduced in the $n \Sigma 13$ interface (seen in Fig. 2). Transitions $C$ and $D$ correspond to $\mathrm{O} 2 p$ to Ti $3 d$ $e_{g}$ and $\mathrm{O} 2 p$ to $\mathrm{Sr} 4 d$ excitations, respectively. Peak $D$ also has contributions from $\mathrm{O} 2 p \rightarrow \mathrm{Sr} 4 d$ transitions since the $\mathrm{Sr}$ $4 d$ DOS becomes large at energies between 12 and $15 \mathrm{eV}$ above the top of the valence band. Peak $C$ is present in the interfacial interband transitions of the $\Sigma 5$ interface but peak $B$ is not observed, while both $B$ and $C$ peaks are dramatically reduced in the $\mathrm{n} \Sigma 13$ interface. At energies of $16.5 \mathrm{eV}$ and $20.1 \mathrm{eV}$, the features $E_{1}$ and $E_{2}$ of interband transitions show up clearly the $\mathrm{Sr} 4 p \rightarrow \mathrm{Ti} 3 d t_{2 g}$ and $e_{g}$ excitations in $\Sigma 5$ boundary while only shoulder-like shapes of the two excitations are seen in $n \Sigma 13$ boundary. Due to the experimental energy resolution, a spin-orbit splitting of the $\mathrm{Sr} 4 p$ level by $0.6 \mathrm{eV},{ }^{60}$ i.e., splitting within $E_{1}$ and $E_{2}$, is not observed in our VEELS measurements. For final states with energies higher than about $15 \mathrm{eV}$ above the Fermi energy, the density of states shows a wealth of different overlapping energy levels that result in broad energy bands. ${ }^{33}$ The transition peaks $F_{1}, F_{2}, G, H$ at transition energies above $24 \mathrm{eV}$ clearly appear in both $\Sigma 5$ and $n \Sigma 13$ boundaries but with lower strength when compared to bulk $\mathrm{SrTiO}_{3}$ with higher intensity. Features of $E_{3}$ at $21.7 \mathrm{eV}$ and $E_{4}$ at $24.2 \mathrm{eV}$ for transitions of $\mathrm{Sr} 4 p$ to Ti $3 d t_{2 g}$ and $e_{g}$ observed on $J_{c v}$ spectra of bulk $\mathrm{SrTiO}_{3}$ were not observable at the $\Sigma 5$ and the $\mathrm{n} \Sigma 13 \mathrm{GBs}$ in this work, again due to energy resolution limits. Peaks $F_{1}$ and $F_{2}$ energetically correspond to the transition of $\mathrm{O} 2 \mathrm{~s}$ to $\mathrm{Ti} 3 d$ and $\mathrm{Sr} 4 d$ levels. Because the initial states of these transitions arise at different atomic sites than the final states, such transitions do not violate selection rules for dipole transitions. Peaks $G$ at $37.5 \mathrm{eV}$ and $H$ at $45.5 \mathrm{eV}$, appearing in the $J_{c v}$ spectra for bulk $\mathrm{SrTiO}_{3}$ as well as in both GB cores, are both due to core-level excitations. Although peak $G$ at $37.5 \mathrm{eV}$ is a weak spectral feature, which is not clearly resolved, it is most likely due to a $\mathrm{Sr} 4 s \rightarrow \mathrm{Ti} 3 d t_{2 g}$ transition, but can also be assigned to the $\operatorname{Sr} N_{1}$ absorption edge. ${ }^{61}$ Also, a Ti $3 p \rightarrow$ Ti $3 d t_{2 g}$ transition is possible since the initial and the final state of this transition belong to different atomic sites. Peak $H$ can be identified with the Ti $M_{2,3}$ edge ${ }^{62}$ and arises from transitions from $\mathrm{Ti} 3 p \rightarrow \mathrm{Ti} 4 s$, Ti $3 d$ and $\mathrm{Sr} 4 d$ conduction bands. The energetic shift of $1.5 \mathrm{eV}$ towards lower energy losses in comparison to the metallic $\mathrm{Ti}$ edge arises from the different oxidation state of $\mathrm{Ti}$ in $\mathrm{SrTiO}_{3}$.

The intensity of the transition peaks, and the integral of the area of the optical properties, are related to the oscillator strength, or $f$, sum rule, and depend on the mass density and the degree of the atomic packing density in the interface, i.e., the interfaces with well ordered and tightly packed atomic structure should, to first order, show the strongest transition strengths, while the complicated $n \Sigma 13$ boundary with many dislocations and a lower packing density and physical density has the weakest interband transitions.

The reduction in intensity of peaks labeled $A, B, C$, and $D$ in the interband transition spectrum of $\Sigma 5$ and $n \Sigma 13 \mathrm{~GB}$ is due to a loss of oxygen and/or a loss of titanium at the GB. Based on previous energy dispersive X-ray measurements, ${ }^{49}$ which have shown an increased $\mathrm{Ti} / \mathrm{Sr}$ atom ratio at the $\mathrm{n \Sigma} 13$ $\mathrm{GB}$, this loss of intensity is due to the formation of oxygen vacancies in the GB region.

The observed reduction in density in the interfacial material and the formation of oxygen vacancies lead to a reduced splitting of the Ti $t_{2 g}$ and Ti $e_{g}$ levels. Because core levels are relatively unaffected by density related changes of bandwidths (e.g., $\mathrm{Sr} 4 p$ levels), transition energies $E_{1}$ and $E_{2}$ remain constant at the GB. Band narrowing effects of the Ti $t_{2 g}$ and Ti $e_{g}$ levels could not be resolved for these transitions. The origin of peaks $F^{\prime}$ and $F$ could not finally be determined. However, peak $F$ shifts by $1.1 \mathrm{eV}$ towards higher energies in the GB region. Finally it can be concluded that the more strongly disturbed $n \Sigma 13$ interface (compared to bulk $\mathrm{SrTiO}_{3}$ and the $\Sigma 5 \mathrm{~GB}$ structure) dramatically changes the electronic structure and therefore optic and electric properties of the GB. For a complete understanding of the electronic structure, calculations of the atomic and the electronic structure would be needed. The explicit influence of segregated Fe to the GB has not been accounted for in this discussion.

\section{B. Optical properties}

The index of refraction decreases gradually into the center of the boundary cores. ${ }^{32}$ For both interface structures, this index contrast corresponds to lower mass density of the interfacial materials arising from changes in the composition and atomic structure compared to the bulk $\mathrm{SrTiO}_{3}$ grains.

Reduced electron densities in both the $\Sigma 5$ and the $n \Sigma 13$ grain boundary cores can be inferred from the reduced integrated intensities and directly from $f$-sum rule calculations. Stronger deviation of the interface structure from the bulk atomic structure, as observed for the $n \sum 13 \mathrm{~GB}$ compared to the $\Sigma 5 \mathrm{~GB}$, leads to a greater difference in the mass density and therefore to a greater difference in the interfacial electron density. Consequently, bulk $\mathrm{SrTiO}_{3}$ exhibits the highest electron density, the $\Sigma 5$ interface intermediate electron densities, and the $n \Sigma 13$ interface the lowest electron density. The order of change in the interfacial electron density correlates with the observed changes in the interfacial index of refraction, so as to give a sense of changes in the physical density and polarizability of the interface. 
To understand the origin of the large changes in interband transition strength within the GB, one should consider that the transition strength is correlated with the physical density of a material, the material's high frequency electronic structure and the polarizability. For example, if either the polarizability or the density decreases, consequently the electron density decreases because the atom number per unit volume decreases with physical density. The relative physical or mass density at the core of the $\Sigma 5 \mathrm{~GB}$ can be estimated on this basis to be $39 \%$, while the density at the $n \Sigma 13$ boundary reduces to $21 \%$ of the bulk density of $\mathrm{SrTiO}_{3}$. Once the electron density reduces with mass density, the electron number participating in the interband transition also decreases. The $\mathrm{n} \Sigma 13$ GB has the smallest physical and especially electron density so that its transition strength is overall reduced compared to the bulk and the $\Sigma 5$ grain boundary (cf. previous results published in Ref. 32).

\section{C. van der Waals-London dispersion interactions and Hamaker coefficients}

The positive value of Hamaker coefficients reveals the existence of attractive van der Waals-London dispersion forces across the GB that are a consequence of the strong optical property gradients. The boundary core serves as an "interfacial layer" of a unique material between the grains, and its optical property discontinuity leads to the attractive van der Waals-London dispersion force. For this interface configuration, the limiting values of the Hamaker coefficient for differing interfacial layer properties would be a maximum for a vacuum interlayer and zero if the interfacial layer (i.e., the grain boundary core) were indistinguishable from bulk $\mathrm{SrTiO}_{3}$. The optical contrast between the bulk grain layers and the grain boundary core determines the magnitude of the Hamaker coefficients. The optical contrast scales inversely with the index of refraction, i.e., 1.00, 1.29, 1.44, and 1.56 for a vacuum interlayer, the $\mathrm{n} \Sigma 13 \mathrm{~GB}$, an $\mathrm{SiO}_{2}$ interlayer, and the $\Sigma 5$ GB, respectively (error bars are \pm 0.05 ). Therefore, the optical contrast is the largest for vacuum, and the smallest for the $\Sigma 5 \mathrm{~GB}$. As a consequence, the Hamaker coefficient as well as the van der Waals-London dispersion force for a vacuum interlayer shows the highest values, followed by the $n \Sigma 13 \mathrm{~GB}$, the $\mathrm{SiO}_{2}$ interlayer. The $\Sigma 5 \mathrm{~GB}$ structure reveals the smallest values.

The important role of the dispersion interaction in GB formation and the final equilibrium GB structure can be seen from the large changes in the dispersion energy comparing free surface energies and interface energies. Forming the $\mathrm{n} \Sigma 13 \mathrm{~GB}$ regains about $69 \mathrm{~mJ} / \mathrm{m}^{2}$ (i.e., $58 \%$ ) of the London dispersion energy in the GB structure (compared to $119 \mathrm{~mJ} / \mathrm{m}^{2}$ ). The $\Sigma 5 \mathrm{~GB}$ regains about $105 \mathrm{~mJ} / \mathrm{m}^{2}$, corresponding to about $88 \%$ of the London dispersion energy of the free surfaces. ${ }^{63,64}$

Although London dispersion energies of $14-24 \mathrm{~mJ} / \mathrm{m}^{2}$ and $44.5-73 \mathrm{~mJ} / \mathrm{m}^{2}$ for the two investigated GB are small, they represent appreciable amounts, between $4 \%$ and up to $10 \%$, of the total GB energies (see Table III). ${ }^{63,64}$ Dispersion forces are usually taken into account when addressing other multiphase systems. Here we have demonstrated that a GB can be considered as just such a multiphase system through changes in materials properties at the atomic level that lead to dispersion interaction caused by the exchange of virtual photons between the two crystalline grains across the grain boundary cores.

\section{Abrupt interfaces vs graded interfaces}

A graded representation of the dielectric properties across even an atomically structured interface approaches its real local properties more closely than by the use of an abrupt and discontinuous model. Numerically different graded layer models give uniform and comparable values of the Hamaker coefficient, whereas the abrupt layer model reveals numerical values that are significantly larger, depending on the essentially arbitrary position of the dielectric discontinuity. For example, the Hamaker coefficient for the $\Sigma 5$ grain boundary based on an abrupt interface model is about $69 \%$ higher than the one based on the quadroid model. The tested graded layer models reveal very similar values for the Hamaker coefficients. The most consistent value of the Hamaker coefficient is obtained for the $\Sigma 5 \mathrm{~GB}$, where the deviation is within $\pm 5 \%$ for different graded response models. For the $n \Sigma 13$ GB, the deviation for different graded response models is within $\pm 16.5 \%$. The same conclusions can be reached also for the absolute value of the van der Waals-London dispersion free energy, as seen in Table III. In this special case of atomically structured grain boundaries with structural changes within one nanometer around the interface plane, this effect demonstrates that the dielectric properties change gradually across an even abrupt interface, but the actual shape of the gradation is not of significant importance due to the very small width of the GB core region (i.e., below $1 \mathrm{~nm})$.

For the graded interface model, the graded interlayer thickness $d$ was chosen to be a natural length scale in the investigated materials, i.e., a Ti-O bond length of $0.195 \mathrm{~nm}$. The experimentally observed width of the gradient in, for instance, the index of refraction, is predominantly determined by the electron probe size $(\approx 0.6 \mathrm{~nm}$ in this study $)$ and the apparent interaction volume, which includes electron beam broadening while penetrating the material and delocalization effects due to the inelastic scattering process. ${ }^{31,40}$ The width of the observed gradient can thus be reduced by using aberration-corrected electron optical lenses providing electron probe sizes of $0.1 \mathrm{~nm}$ or less. ${ }^{65}$ Therefore, the use of a modeled form of the graded dielectric response instead of the experimentally observed gradient (see above) becomes physically more meaningful since no experimental artifacts are imposed on the determined optical properties.

The difference between the graded and the abrupt interface dielectric response models is most clearly seen in Fig. 6. Here the Hamaker coefficient is shown for the abrupt model as well as for the quadroid model as a function of the grain boundary core width $L$ between two symmetric $\mathrm{SrTiO}_{3}$ grain boundaries. The curves cover the $L$ dependence of the Hamaker coefficient from $L=0$ to $L$ appropriate for the $\Sigma 5$ ( $L$ $=0.6 \mathrm{~nm})$ and the $\mathrm{n} \Sigma 13(L=0.9 \mathrm{~nm})$ core widths. ${ }^{32,48,49}$ For large values of $L$ both models will converge since at signifi- 


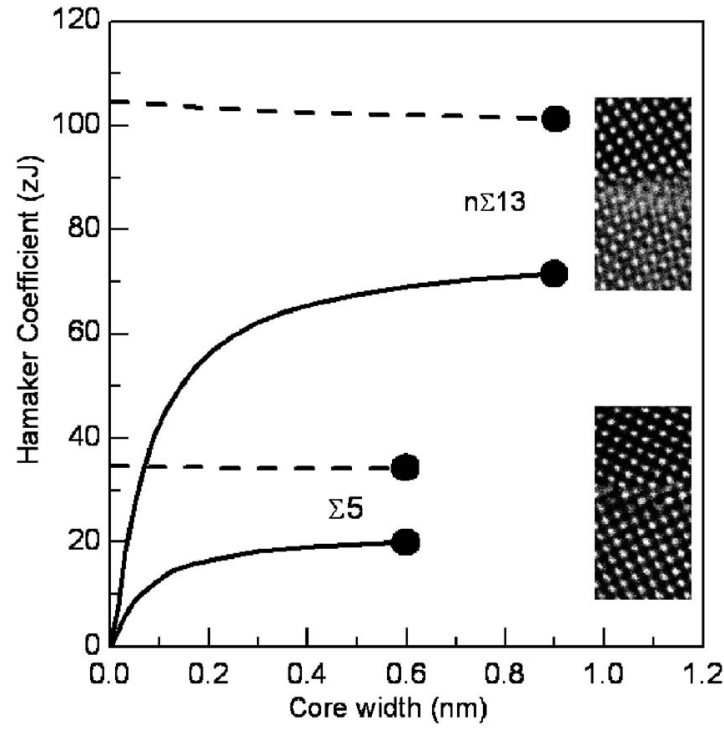

FIG. 6. Hamaker coefficients for the $\Sigma 5$ and the $n \Sigma 13$ grain boundaries. The upper dotted lines correspond to the abrupt dielectric response models while the lower solid lines have been computed using the more realistic quadroid layer model.

cantly large separations with respect to the width of the boundary core, the abrupt model and the graded models become indistinguishable. ${ }^{59}$ However, at the core width of $0.6 \mathrm{~nm}$ and $0.9 \mathrm{~nm}$ the Hamaker coefficients are different from the abrupt interface models.

For graded layer models the Hamaker coefficient becomes zero for $L \rightarrow 0$. For the abrupt spatial dielectric response model, the Hamaker coefficient remains finite and almost constant in the whole range of $L$ values. This anomaly is a simple consequence of the quadroid (as well as any other continuous graded response) model that assumes a continuous dielectric function as well as its derivative across the grain boundary. ${ }^{57}$ In this case, the van der Waals-London dispersion interaction free energy $G(L)$ has a finite limit for $L \rightarrow 0$, which results in a vanishing Hamaker coefficient, following $A(L \rightarrow 0)=12 \pi L^{2} G(L)$. For larger $L$ the graded response Hamaker coefficient increases and eventually saturates after the van der Waals-London dispersion interaction free energy resumes its whole dependence on $L$ for $L$ larger than the thickness of the boundary layers. This behavior of the Hamaker coefficient is a salient feature of the graded, continuous models, and should be taken into account when making numerical estimates of the van der Waals-London dispersion interaction. Clearly the abrupt model unphysically overestimates the interaction at small grain boundary core thicknesses. The origin of the small change in the Hamaker coefficient with $L$ for the abrupt model is the (small) retardation effect that leads to a different $L$-dependence of the interaction free energy:

$$
G(L) \propto L^{-n}, \quad \text { with } n>2 \text { for large values of } L \text { (Ref. 57). }
$$

For the separations under consideration in this study, retardation effects can be neglected. Retardation effects are also negligible in the graded response calculations since the width of the diffuse boundary layers is significantly smaller than any characteristic length scales concerning retardation effects.

As $L$ becomes smaller than the Ti-O bond length, i.e., the thickness of the diffuse boundary in the graded response model, the Lifshitz formulation of the macroscopic electrodynamics on which the van der Waals-London interaction is based, breaks down. In such a case, one would have to resort to an atomic calculation and evaluate all the dispersion interactions between single atoms separately. But even if this exercise can be accomplished, problems would remain in assessing when, and indeed if ever, the higher order nonpairwise components of the dispersion interactions, which are non-negligible and strong in dense systems, can be neglected. Such conditions are very computing time consuming and the results would still remain far from being exact.

\section{CONCLUSION}

Electronic structure investigations for $\Sigma 5$ and $n \Sigma 13$ GB in $\mathrm{SrTiO}_{3}$ were performed by Kramers-Kronig analyses of previously collected spatially resolved VEELS data. The experimentally determined interband transition energies for bulk $\mathrm{SrTiO}_{3}$ and both $\Sigma 5$ and $\mathrm{n} \Sigma 13$ GB structures were evaluated and assigned to distinct interband transitions by comparison with calculated densities of states. The acquired VEELS line scans show a decrease in intensity in the interband transition strength that is due to a reduced electron density within the GB compared to bulk $\mathrm{SrTiO}_{3}$. The index of refraction exhibits big gradient contrast across both grain boundary structures, i.e., the index of refraction remains almost the same on the surface of grains, but decreases gradually if away from grain edges and stretching into the centre of the GB. The index of refraction reaches the lowest value at the interface core of the GB.

In both $\Sigma 5$ and $n \Sigma 13$ GB regions, the interband transition strength showed a GB specific line shape and therefore a modified electronic structure compared with the bulk. Decreasing transition strengths for $\mathrm{O} 2 p$ to metal $d$ transitions reconfirm an enrichment of oxygen vacancies inside the grain boundary cores. Moreover, the Ti atoms in the GB are reduced in their nominal oxidation state as indicated by a negative chemical shift in the EELS spectrum ${ }^{31}$ as well as the loss of the transition peak from $\mathrm{O} 2 p$ to $\mathrm{Ti} 3 d t_{2 g}$ and dramatically reduced strength involving the interband transition in the Ti energy levels.

An observed reduction of the index of refraction and electron density inside both interfaces is due to a smaller mass density and, therefore, a smaller electron density in the GB cores when compared to the bulk materials. Because of the presence of these graded contrasts of electronic properties across the GB, which are the fundamental origin of the longrange van der Waals-London dispersion interaction, we were able to propose several new graded layer dielectric response models based on quadratic, circuloid, and quadroid functions, in order to calculate the Hamaker coefficient and the van der Waals-London dispersion free energies of the two investigated grain boundary structures. The abrupt-layer and 
linear layer models, both exhibiting discontinuities either in the value or the derivative, or both, of the dielectric properties, give rise to physical values of the Hamaker coefficient. However, Hamaker coefficients calculated from the three proposed graded layer dielectric response models (quadratic, circuloid, and quadroid), for the same interlayer materials, show much less variation in their numerical values, indicating that they represent the interfaces more realistically.

The determination of quantitative physical properties and interfacial electronic structure of GB at high spatial resolution goes beyond previous interface studies that focused on atomic structural or compositional observations of interfaces. A combination of this technique with well established routes for atomic structure and composition analyses gives a deeper fundamental understanding of formation processes. Furthermore, it sheds light on how certain GB structures and micro- structures are established, and how their properties are optimized to produce unique functional materials with desirable properties and performance.

\section{ACKNOWLEDGMENTS}

The authors acknowledge stimulating discussions with R.M. Cannon (1943-2006) and M. Rühle. K.v.B. acknowledges S.J. Pennycook and financial support from the Alexander-von-Humboldt Foundation and from Oak Ridge Associated Universities. This work was partially supported by the European Union under Contract No. G5RD-CT-200100586 and by NSF No.DMR-0010062. Financial support for R.P. is in part provided by the European Commission under Contract No. NMP3-CT-2005-013862 (INCEMS).
${ }^{1}$ M. Tang, W. C. Carter, and R. M. Cannon, Phys. Rev. B 73, 024102 (2006).

${ }^{2}$ C. M. Bishop, W. C. Carter, and R. M. Cannon, Acta Mater. 53, 4755 (2005).

${ }^{3}$ H. Gu, R. M. Cannon, and M. Rühle, J. Mater. Res. 13, 476 (1998).

${ }^{4}$ Y.-M. Chiang, J.-R. Lee, H. Wang, Microstructure and Intergranular Phase Distribution in Bi2O3-Doped $\mathrm{ZnO}$, Ceramic Microstructure: Control at the Atomic Level, edited by A. P. Tomsia and A. Glaeser (Plenum Press, New York, 1998), pp. 131147.

${ }^{5}$ H. Gu, X. Pan, R. M. Cannon, and M. Rühle, J. Am. Ceram. Soc. 81, 3125 (1998).

${ }^{6}$ R. M. Cannon, M. Rühle, M. J. Hoffmann, R. H. French, H. Gu, A. P. Tomsia, and E. Saiz, in Grain Boundary Engineering in Ceramics, edited by T. Sakuma, L. Sheppard, and Y. Ikuhara (Ceramic Transactions, American Ceramic Society, Columbus, Ohio), Vol. 118, 427, (2000).

${ }^{7}$ D. Dimos, P. Chaudhari, and J. Mannhart, Phys. Rev. B 41, 4038 (1990)

${ }^{8}$ W. Heywang, J. Am. Ceram. Soc. 47, 484 (1964).

${ }^{9}$ T. K. Gupta, J. Am. Ceram. Soc. 73, 1817 (1990).

${ }^{10}$ D. Dimos, P. Chaudhari, and J. Mannhart, Phys. Rev. B 41, 4038 (1990)

${ }^{11}$ E. Y. Sun, P. F. Becher, K. P. Plucknett, C.-H. Hsueh, K. B. Alexander, S. B. Waters, K. Hirao, and M. E. Brito, J. Am. Ceram. Soc. 81, 2831 (1998).

${ }^{12}$ N. P. Padture, J. Am. Ceram. Soc. 77, 519 (1994).

${ }^{13}$ J. Cao, W. J. MoberlyChan, L. C. DeJonghe, C. J. Gilbert, and R. O. Ritchie, J. Am. Ceram. Soc. 79, 461 (1996).

${ }^{14}$ V. Ravikumar, R. P. Rodrigues, and V. P. Dravid, J. Phys. D 29, 1799 (1996).

${ }^{15}$ N. D. Browning, J. P. Buban, H. O. Moltali, and S. J. Pennycook, Appl. Phys. Lett. 74, 2638 (1999).

${ }^{16}$ D. A. Crandles, B. Nicholes, C. Dreher, C. C. Homes, A. W. McConnell, B. P. Clayman, W. H. Gong, and J. E. Greedan, Phys. Rev. B 59, 12842 (1999).

${ }^{17}$ M. Leonhardt, J. Jamnik, and J. Maier, Electrochem. Solid-State Lett. 2, 333 (1999).
${ }^{18}$ H.-J. Kleebe, J. Ceram. Soc. Jpn. 105, 453 (1997).

${ }^{19}$ Q. Jin, D. S. Wilkinson, and G. C. Weatherly, J. Am. Ceram. Soc. 82, 1492 (1999).

${ }^{20}$ Y.-M. Chiang, L. A. Silverman, R. H. French, and R. M. Cannon, J. Am. Ceram. Soc. 77, 1143 (1994).

${ }^{21}$ J. P. Gambino, W. D. Kingery, G. E. Pike, and H. R. Phillip, J. Am. Ceram. Soc. 72, 642 (1989).

${ }^{22}$ N. Hirosaki et al., J. Ceram. Soc. Jpn. 104, 50 (1996).

${ }^{23}$ J. Luo, H. Wang, and Y.-M. Chiang, J. Am. Ceram. Soc. 82, 916 (1999).

${ }^{24}$ A. P. Sutton and R. W. Balluffi, Interfaces in Crystalline Materials (Oxford University Press, New York, 1995).

${ }^{25}$ Z. L. Wang, Micron 27, 265 (1996).

${ }^{26}$ A. D. Dorneich, R. H. French, H. Müllejans, S. Loughin, and M. Rühle, J. Microsc. 191, 286 (1998).

${ }^{27}$ R. H. French, H. Müllejans, and D. J. Jones, J. Am. Ceram. Soc. 81, 2549 (1998).

${ }^{28}$ L. Ryen, X. Wang, U. Helmersson, and E. Olsson, J. Appl. Phys. 85, 2828 (1999).

${ }^{29}$ L. Normand and A. Thorel, Electron Microscopy 1998, Proceedings of ICEM-14, Cancun, Mexico (1998) 625.

${ }^{30}$ R. H. French, H. Müllejans, D. J. Jones, G. Duscher, R. M. Cannon, and M. Rühle, Acta Mater. 46, 2271 (1998).

${ }^{31}$ K. van Benthem, R. H. French, W. Sigle, C. Elsässer, and M. Rühle, Ultramicroscopy 86, 303 (2001).

${ }^{32}$ K. van Benthem, G. Tan, L. K. DeNoyer, R. H. French, and M. Rühle, Phys. Rev. Lett. 93, 227201 (2004).

${ }^{33}$ K. van Benthem, C. Elsässer, and R. H. French, J. Appl. Phys. 90, 6156 (2001).

${ }^{34}$ A. C. Maggs and N. W. Ashcroft, Phys. Rev. Lett. 59, 113 (1987).

${ }^{35}$ M. Dion, H. Rydberg, E. Schroeder, D. C. Langreth, and B. L. Lundqvist, Phys. Rev. Lett. 92, 246401 (2004).

${ }^{36}$ O. A. von Lilienfeld, I. Tavernelli, U. Rothlisberger, and D. Sebastiani, Phys. Rev. Lett. 93, 153004 (2004).

${ }^{37}$ R. H. French, J. Am. Ceram. Soc. 83, 2117 (2000).

${ }^{38}$ H. C. Hamaker, Physica (The Hague) 4, 1058 (1937).

${ }^{39}$ A. Strecker, U. Salzberger, and J. Mayer, Prakt. Metallogr. 30, 482 (1993).

${ }^{40}$ R. F. Egerton, Electron Energy-Loss Spectroscopy in the Electron 
Microscope, 2nd edition (Plenum Press, New York and London, 1996).

${ }^{41}$ M. Cardona, Phys. Rev. 140, A651 (1965).

${ }^{42}$ R. Waser, T. Baiatu, and K.-H. Härtl, J. Am. Ceram. Soc. 73, 1645 (1990).

${ }^{43}$ R. Waser, T. Baiatu, and K.-H. Härtl, J. Am. Ceram. Soc. 73, 1654 (1990).

${ }^{44}$ R. Waser, T. Baiatu, and K.-H. Härtl, J. Am. Ceram. Soc. 73, 1663 (1990).

${ }^{45}$ H. Müllejans and R. H. French, Microsc. Microanal. 6, 297 (2000).

${ }^{46}$ The electronic Structure Tools (EST) consists of a number of programs for the quantitative analysis of optical, VUV and EELS spectra. It has been developed under Grams, a PC based spectroscopy environment. EST is available from Deconvolution and Entropy Consulting, 755 Snyder Hill Road, Ithaca NY 14850, or http://www.deconvolution.com

${ }^{47}$ D. Y. Smith, Dispersion Theory, Sum Rules, and Their Application to the Analysis of Optical Data in Handbook of Optical Constants of Solids edited by E. D. Palik (Academic Press, New York, 1985), pp. 35-68.

${ }^{48}$ F. Ernst, O. Kienzle, and M. Rühle, J. Eur. Ceram. Soc. 19, 665 (1999).

${ }^{49}$ S. Hutt, O. Kienzle, F. Ernst, and M. Rühle, Z. Metallkd. 92, 2 (2001).

${ }^{50}$ E. M. Lifshitz, Sov. Phys. JETP 2, 73 (1956).

${ }^{51}$ I. E. Dzyaloshinskii, E. M. Lifshitz, and L. P. Pitaevskii, Adv. Phys. 10, 165 (1961).

${ }^{52}$ B. W. Ninham and V. A. Parsegian, J. Chem. Phys. 52, 4578 (1970).
${ }^{53}$ V. Adrian Parsegian, van der Waals Forces, A Handbook for Biologists, Chemists, Engineers, and Physicists (Cambridge University Press, Cambridge, 2005).

${ }^{54}$ R. Podgornik, R. H. French, and V. A. Parsegian, J. Chem. Phys. 124, 044709 (2006). Full spectral Hamaker coefficients can be calculated using the add-a-layer method using the open source program Gecko Hamaker, available at http://sourceforge.net/ projects/geckoproj

${ }^{55}$ G. L. Tan, M. F. Lemon, R. H. French, and D. J. Jones, Phys. Rev. B 72, 205117 (2005).

${ }^{56}$ D. A. G. Bruggemann, Ann. Phys. 24, 636 (1935).

${ }^{57}$ N. Maron and O. Maron, Mon. Not. R. Astron. Soc. 357, 873 (2005).

${ }^{58}$ N. D. Browning and S. J. Pennycook, J. Phys. D 29, 1779 (1996).

${ }^{59}$ R. Podgornik and V. A. Parsegian, J. Chem. Phys. 121, 7467 (2004).

${ }^{60}$ D. Bäuerle, W. Braun, V. Saile, G. Sprüssel et al., Z. Phys. B 29, 179 (1978).

${ }^{61}$ Y.-N. Xu, W. Y. Ching, and R. H. French, Ferroelectrics 111, 23 (1990).

${ }^{62}$ C. C. Ahn and O. L. Krivanek, EELS Atlas, A reference collection of electron energy loss spectra covering all stable elements (Gatan Inc., Pleasanton, CA, 1983).

${ }^{63}$ J. Padilla and D. Vanderbilt, Surf. Sci. 418, 64 (1998).

${ }^{64}$ T. Ochs, S. Köstlmeier, and C. Elsässer, Integr. Ferroelectr. 32, 959 (2001).

${ }^{65}$ M. Varela, A. R. Lupini, K. van Benthem, A. Y. Borisevich, M. F. Chisholm, N. Shibata, E. Abe, and S. J. Pennycook, Annu. Rev. Mater. Res. 35, 539 (2005). 